\title{
Dislipidemia y relación con enfermedad tiroidea en agricultores de la zona cafetera
}

\section{Dyslipidemia and relationship with thyroid disease in farmers in the Colombian coffee growing area}

\author{
Patricia Landázuri, Ángela Liliana Londoño-Franco, Beatriz Restrepo-Cortés, \\ Adolfo león Bayona- Zorro, Juan Farid Sánchez-López • Armenia (Colombia)
}

DOI: https://doi.org/10.36104/amc.2019.1094

\section{Resumen}

Introducción: tiroxina libre (T4L), triyodo tironina libre (T3L) y hormona estimulante del tiroides (TSH), se reconocen como reguladoras de síntesis, movilización y degradación de lípidos.

Objetivo: encontrar una relación entre dislipidemias y enfermedad tiroidea en una población adulta.

Métodos: este fue un estudio de corte transversal, en 819 individuos. Se midieron TSH, T4L, colesterol total (CT), colesterol en lipoproteínas de baja densidad (C-LDL), colesterol en lipoproteínas de alta densidad (C-HDL) y triglicéridos (TG).

Resultados: la población general mostró CT, C-LDL normales, C-HDL bajo y TG elevados. La prevalencia de dislipidemia fue: CT elevado $31.9 \%, \mathrm{C}-\mathrm{HDL}<40 \mathrm{mg} / \mathrm{dL} 57.9 \%$. C-LDL alto $24.3 \%$ y TG $\geq 200 \mathrm{mg} / \mathrm{dL}, 26 \%$. La frecuencia de TSH $\geq 4.6 \mathrm{mIU} / \mathrm{L}$ fue $8.1 \%$. La prevalencia de hipotiroidismo manifiesto fue de $1.2 \%$ y la de hipotiroidismo subclínico de $6.7 \%$. La prevalencia de CT elevado en individuos con hipotiroidismo manifiesto y en normales fue de $50.0 \%$ y $31.6 \%$ respectivamente. Mientras en hipotiroidismo subclínico fue de $43.6 \%$ y $31.0 \%$ p: 0.04 para CT (elevado); $40.0 \%$ y $23.2 \%$ p: 0.006 para C-LDL elevado.

Se encontró relación significativa entre TSH $\geq 4.6 \mathrm{mIU} / \mathrm{L}$ con CT elevado (OR 1.9 p 0.01 ) y CLDL elevada (OR 2.5 p: 0.001); la frecuencia de C-LDL elevado fue de 2.2 veces en personas con hipotiroidismo subclínico con relación a personas con niveles normales de TSH.

Conclusión: en los trabajadores del agro, por sus particulares condiciones socioeconómicas y de acceso al sistema de salud, es importante diseñar programas de tamizaje de biomarcadores que permitan avizorar de manera precoz cambios en las hormonas tiroideas, TSH y perfil lipídico, para realizar intervenciones de prevención secundaria que contribuyan a la disminución de la morbimortalidad por eventos cardiovasculares. (Acta Med Colomb 2019; 44. DOI: https://doi. org/10.36104/amc.2019.1094).

Palabras clave: hipotiroidismo, dislipidemias, agricultores, hormonas tiroideas

\section{Abstract}

Introduction: free thyroxine (free T4 ), free triiodothyronine (free T3) and thyroid stimulating hormone (TSH), are recognized as regulators of synthesis, mobilization and lipid degradation.

Objective: to find a relationship between dyslipidemias and thyroid disease in an adult population.

Methods: cross-sectional study in 819 individuals, farmers in the coffee zone, Quindío, Colombia. TSH, free T4, total cholesterol (TC), low density lipoprotein cholesterol (LDL-C), high density lipoprotein cholesterol (HDL-C) and triglycerides (TG) were measured.

Results: the general population showed normal TC and LDL-C, low HDL-C and elevated TG. The prevalence of dyslipidemia was: elevated TC 31.9\%, HDL-C <40 mg / dL 57.9\%, highLDL-C $24.3 \%$ and $\mathrm{TG} \geq 200 \mathrm{mg} / \mathrm{dL} 26 \%$. The frequency of $\mathrm{TSH} \geq 4.6 \mathrm{mIU} / \mathrm{L}$ was $8.1 \%$. The prevalence of overt hypothyroidism was $1.2 \%$ and that of subclinical hypothyroidism $6.7 \%$. The prevalence of high TC in individuals with overt hypothyroidism and normal ones was between 50 and $31.6 \%$ respectively, while in subclinical hypothyroidism it was between 43.6 and $31 \%$ (p: 0.04 for elevated TC); 40 and $23.2 \%$ p: 0.006 for high LDL-C.
Patricia Landázuri: Doctora en Ciencias Biológicas, Magister en Biología, Líder Grupo de Investigación en Enfermedades Cardiovasculares y Metabólicas-GECAVYME; Dra. Ángela Liliana Londoño-Franco: Especialista en Epidemiología, Doctora en Medicina Preventiva y Salud Pública, Docente Investigadora, Líder Grupo de Investigación en Salud Pública; Dra. Beatriz Restrepo-Cortés: Doctora en Fisiopatología Celular Molecular y sus Implicaciones Farmacológicas. Magister en Biología; Dr. Adolfo León Bayona-Zorro: Especialista en Medicina Interna y Endocrinología; Dr. Juan Farid Sánchez-López: Doctor en Medicina Preventiva y Salud Pública, Magister en Salud Pública, Médico Especialista en Salud Ocupacional. Facultad Ciencias de la Salud, Universidad del Quindío. Armenia (Colombia)

Correspondencia: Dra. Ángela Liliana Londoño-Franco. Armenia (Colombia).

E-mail: angelalilianal@uniquindio.edu.co Recibido: 8/IX/2017 Aceptado: 22/VII/2019 
A significant relationship between TSH $\geq 4.6 \mathrm{mIU} / \mathrm{L}$ with high TC (OR $1.9 \mathrm{p} 0.01)$ and high LDL-C (OR 2.5 p: 0.001) was found; the frequency of elevated LDL-C was 2.2 times higher in people with subclinical hypothyroidism than in people with normal levels of TSH.

Conclusion: in agricultural workers, due to their particular socio-economic conditions and access to the health system, it is important to design biomarker screening programs that allow early detection of changes in thyroid hormones, TSH and lipid profile, to perform interventions of secondary prevention that contribute to the decrease in morbidity and mortality due to cardiovascular events.

(Acta Med Colomb 2019; 44. DOI: https://doi.org/10.36104/amc.2019.1094).

Keywords: hypothyroidism, dyslipidemias, farmers, thyroid hormones.

\section{Introducción}

Las hormonas tiroideas han sido reconocidas como hormonas que regulan diversos procesos del metabolismo, incluyendo la síntesis, movilización y degradación de lípidos $(1,2)$. Una de las causas de enfermedad cardiovascular es la disfunción de las hormonas tiroideas: se estima que entre el 1.3 y $12 \%$ de los pacientes con hipercolesterolemia tienen hipotiroidismo (3, 4), Estos estudios reportan riesgo aumentado de dislipidemias asociado con enfermedad cardiovascular aterosclerótica. Así, se demuestra que los pacientes con hipotiroidismo manifiesto muestran niveles consistentemente elevados de colesterol y triglicéridos (TG), mientras los pacientes hipertiroideos muestran niveles reducidos de lípidos (1, 4). También algunos trabajos han mostrado alteraciones de los lípidos en el hipertiroidismo subclínico, sugiriendo que las hormonas tiroideas, y la hormona estimulante de la tiroides (TSH) ejercen efectos independientes sobre el metabolismo lipídico (5-7).

Respecto a los mecanismos de acción de las hormonas tiroideas y TSH sobre los lípidos, avances en biología molecular han demostrado que pueden actuar a través de inducir la expresión de la 3-hidroxi-3-metil-glutaril-CoA reductasa (HMG-CoA), del receptor de lipoproteínas de baja densidad (LDLR), la lipasa, la proteína de transferencia de ésteres de colesterol (CETP) y otras enzimas y moléculas involucradas como factores de riesgo cardiovascular (8-12). También se describió que la TSH induce la adipogénesis (13), lipólisis (14) y aumentan la actividad de la HMG-CoA (15).

Dado que el hipotiroidismo y el hipertiroidismo son condiciones más comunes en la población general, la relación entre las hormonas tiroideas y el perfil lipídico en la población eutiroidea ha generado mucho interés (4, 16 ), porque pueden generar conocimiento para la prevención de la enfermedad tiroidea en esta población. Aunque varios estudios han revelado que la tiroxina libre (T4L), la triyodotiroxina libre (T3) y la TSH se asocian significativamente con el perfil lipídico independientemente del sexo $(17,18)$, en la mayoría de ellos, T4, T3 y TSH no se han medido juntas $(19,20)$. Por lo tanto, no es posible excluir los efectos de la interacción entre T3, T4 y TSH en asociación con el perfil lipídico. Sin embargo, hay una creciente cantidad de evidencia que indica que esta asociación es parcialmente aportada por el efecto directo de la TSH sobre el perfil lipídico y también asociada con síndrome metabólico (21-23).

Finalmente aunque la alteración en el perfil lipídico es una observación común en pacientes con disfunción tiroidea, el conocimiento actual sobre la relación entre los lípidos y hormonas tiroideas y THS juntas es insuficiente, razón por la cual el objetivo de este trabajo fue encontrar la relación entre dislipidemias y enfermedad tiroidea en una población adulta de zonas rulares del departamento del Quindío.

\section{Material y métodos}

Se realizó un estudio de corte transversal, en población adulta residente en área rural de ocho municipios del departamento del Quindío. Se calculó una muestra representativa de la población agrícola, en total se incluyeron 819 participantes para el análisis. Criterio de inclusión: sin diagnóstico previo de enfermedad tiroidea; criterio de exclusión: pruebas diagnósticas o terapéuticas previas con medios de contraste yodados.

\section{Parámetros antropométricos y bioquímicos}

Muestras sanguíneas. Se obtuvieron después de $12 \mathrm{~h}$ de ayuno por punción venosa en 2 tubos secos. El suero se obtuvo por centrifugación a $1.000 \mathrm{~g}$ por $15 \mathrm{~min}$, a $4^{\circ} \mathrm{C}$, separado en microtubos de $1.5 \mathrm{~mL}$ y almacenado a $-20^{\circ} \mathrm{C}$ hasta su uso.

Pruebas tiroideas. La cuantificación de la TSH y T4L se hicieron por medio del kit comercial (Novatec Immunodiagnóstica $G M B H$ ), de acuerdo con las instrucciones del fabricante y se leyó en un equipo lector de ELISA Stat Fax 303/Plus, en una longitud de onda $450 \mathrm{~nm}$. Los valores de referencia considerados normales fueron 0.3-4.5 mIU/L para TSH, y 8-20 ng/L para T4 libre. Se clasificó el hipotiroidismo en hipotiroidismo manifiesto (TSH alta y $\mathrm{T}_{4}$ baja) e hipotiroidismo subclínico ( $\mathrm{TSH}$ alta y $\mathrm{T}_{4} \mathrm{~L}$ normal)

Perfil lipídico. El colesterol total, colesterol HDL y los triglicéridos se midieron por métodos enzimáticos comerciales (HUMAN®) y siguiendo las recomendaciones del fabricante. Para quienes tuvieron los triglicéridos inferiores a $400 \mathrm{mg} / \mathrm{dL}$, el colesterol en las lipoproteínas de baja densidad (C-LDL) se calcularon con la ecuación de Friedewald (24). 
Índice de masa corporal. Se calculó como $\left(\mathrm{Kg} / \mathrm{m}^{2}\right)$.

Análisis estadístico. Se procesaron y analizaron datos en SPSS versión 19. Se realizó análisis descriptivo y bivariado, para la distribución de Ji cuadrado se asumió significancia de $<0.05$. Se realizó test de Kolmogorov- Smirnov para variables cuantitativas y con el resultado se decidió la utilización de técnicas paramétricas o no paramétricas; para las variables de distribución normal se aplicó el test de T Student. Se buscó correlación de las cifras de lípidos con edad, número de cigarrillos al día, tiempo fumando y nivel educativo. Se realizó análisis multivariado de regresión logística para buscar un modelo de variables relacionadas con dislipidemias.

\section{Resultados}

Se incluyeron 819 agricultores, la media de edad de los participantes fue de 48.14 años (IC 95\%: 47.13-49.14) con un mínimo de 18 y un máximo de 87 . La mayoría fueron hombres (58.7\%). El promedio de años de escolaridad fue de 5.94 (IC 95\%: 5.66-6.22), del total un 67.5\% estudiaron menos de 8 años, $21.6 \%$ eran analfabetas o tenían primero de primaria (Tabla 1).

$\mathrm{Al}$ indagar por antecedentes médicos se encontró que solo cuatro personas referían sufrir de "colesterol o triglicéridos altos" y en la historia de medicamentos recibidos, seis personas estaban en tratamiento para dislipidemia. En la población total se observaron promedios dentro de límites normales para colesterol total $(184.6 \mathrm{mg} / \mathrm{dL})$ y colesterol LDL (C-LDL) (110.1 mg/dL), bajos para colesterol HDL $(39.5 \mathrm{mg} / \mathrm{dL})$ y elevados para triglicéridos $(174.7 \mathrm{mg} /$
Tabla 1. Características de la Población de estudio.

\begin{tabular}{|c|c|c|c|}
\hline \multirow[t]{2}{*}{ Variable } & \multirow[t]{2}{*}{ Valores } & \multicolumn{2}{|c|}{ Frecuencia } \\
\hline & & $\mathbf{N}^{o}$ & $\%$ \\
\hline \multirow[t]{2}{*}{ Sexo } & Masculino & 481 & 58.7 \\
\hline & Femenino & 338 & 41.3 \\
\hline \multirow[t]{2}{*}{ Edad en años } & $<45$ & 319 & 39.4 \\
\hline & $45 y>$ & 490 & 60.6 \\
\hline \multirow[t]{4}{*}{ IMC $\left(\mathrm{kg} / \mathrm{m}^{2}\right)$} & 30 o más & 110 & 13.4 \\
\hline & 25.1-29.9 & 283 & 34.6 \\
\hline & $20-25$ & 343 & 41.9 \\
\hline & $<20$ & 83 & 10.1 \\
\hline \multirow[t]{4}{*}{ Escolaridad } & Ninguna & 71 & 8.9 \\
\hline & $\begin{array}{l}1 \text { año de } \\
\text { primaria }\end{array}$ & 30 & 3.8 \\
\hline & $<8$ años & 539 & 67.5 \\
\hline & 8 y más & 260 & 32.5 \\
\hline \multirow[t]{5}{*}{ Estrato } & 0 & 15 & 2.0 \\
\hline & 1 & 451 & 59.6 \\
\hline & 2 & 265 & 35.0 \\
\hline & 3 & 24 & 3.2 \\
\hline & 5 & 2 & 0.3 \\
\hline
\end{tabular}

Tabla 2. Cifras de lípidos totales y según edad-sexo.

\begin{tabular}{|c|c|c|c|c|c|c|c|}
\hline \multirow[b]{3}{*}{$\mathrm{CT} \mathbf{m g} / \mathrm{dL}^{\mathrm{a}}$} & \multicolumn{5}{|c|}{ Medidas tendencia central } & \multicolumn{2}{|c|}{ Diferencia promedios } \\
\hline & & Promedio & IC $95 \%$ & Min. & Máx. & $\mathbf{p}$ & IC $95 \%$ \\
\hline & $\begin{array}{l}\text { Población total } \\
\text { Edad grupos } \geq 45 /<45 \\
\text { Masculino/femenino }\end{array}$ & $\begin{array}{c}184.6 \\
193.8 / 170.3 \\
183.1 / 186.7\end{array}$ & $179.9-189.3$ & 88.6 & 1758 & $\begin{array}{l}\mathbf{0 . 0 0 0} \\
0.47\end{array}$ & $\begin{array}{ll}13.8 & 33.1 \\
-13.2 & 6.0\end{array}$ \\
\hline C-HDL mg/dL ${ }^{b}$ & $\begin{array}{l}\text { Población total } \\
\text { Edad grupos } \geq 45 /<45 \\
\text { Masculino/femenino }\end{array}$ & $\begin{array}{c}39.5 \\
40.1 / 38.7 \\
38.2 / 41.3\end{array}$ & $38.8-40.2$ & 12.9 & 77.8 & $\begin{array}{c}0.07 \\
\mathbf{0 . 0 0 0}\end{array}$ & $\begin{array}{ll}-0.1 & 2.8 \\
-4.5 & -1.6\end{array}$ \\
\hline C-LDLmg/dL ${ }^{\mathrm{c}}$ & $\begin{array}{l}\text { Población total } \\
\text { Edad grupos } \geq 45 /<45 \\
\text { Masculino/femenino }\end{array}$ & $\begin{array}{c}110.1 \\
115.0 / 102.3 \\
109.7 / 110.8\end{array}$ & 107.6-112.6 & 25.4 & 358.5 & $\begin{array}{l}\mathbf{0 . 0 0 0} \\
0.67\end{array}$ & $\begin{array}{rr}7.6 & 17.8 \\
-6.1 & 4.0\end{array}$ \\
\hline C-VLDL mg/dL & Población total & 30.6 & $29.7-31.6$ & 7.4 & 79.4 & & \\
\hline $\mathrm{TG} \mathbf{m g} / \mathrm{dL}^{\mathrm{d}}$ & $\begin{array}{l}\text { Población total } \\
\text { Edad grupos } \geq 45 /<45 \\
\text { Masculino/femenino }\end{array}$ & $\begin{array}{c}174.7 \\
185.1 / 157.3 \\
176.9-171.7\end{array}$ & $161.6-187.8$ & 36.8 & 4160 & $\begin{array}{l}\mathbf{0 . 0 4} \\
0.7\end{array}$ & $\begin{array}{rr}0.9 & 54.6 \\
-19.7 & 30.1\end{array}$ \\
\hline
\end{tabular}


dL). Los promedios de colesterol total y de C-LDL fueron significativamente mayores en las personas de 45 y más años $(p=0.000)$, mientras que el C-HDL fue mayor en los hombres $(p=0.000)$ (Tabla 2).

No se encontró correlación de las cifras de lípidos con edad, número de cigarrillos al día, tiempo fumando o nivel educativo.

En la población total la prevalencia de alteraciones en el perfil lipídico fue la siguiente: colesterol total elevado $31.9 \%$, C-HDL menor a 40mg/dL 57.9\%. C-LDL elevado en toda la población un $24.3 \%$ (n 199), con niveles entre 130 y $159 \mathrm{mg} / \mathrm{dL}$, un $17.3 \%$ (n 141), entre 160 y $189 \mathrm{mg} / \mathrm{dL}$ un $7.2 \%$ (n 59) y por encima de $190 \mathrm{mg} / \mathrm{dL}$ un $1.7 \%$ (n 14). Los triglicéridos tuvieron valores de $200 \mathrm{mg} / \mathrm{dL}$ o mayores en un 26\% (n 213), un $24.3 \%$ con niveles entre 200 y 499 $\mathrm{mg} / \mathrm{dL}$ y un $1.7 \%$ con niveles de $500 \mathrm{mg} / \mathrm{dL}$ o mayores.

$\mathrm{Al}$ explorar en el análisis bivariado la relación entre niveles alterados de lípidos con variables de riesgo incluidas se encontró asociación no causal entre edad de 45 y más años con CT mayor o igual a $200(\mathrm{OR} 2.1 \mathrm{p}=0.000), \mathrm{C}-\mathrm{HDL}$ menor a $40(\mathrm{OR} 1.6 \mathrm{p}=0.001)$ y $\mathrm{C}-\mathrm{LDL}$ mayor o igual a
130 (OR $1.8 \mathrm{p}=0.001)$. Una escolaridad menor a ocho años se asoció con CT y C-LDL elevados (OR $2.1 \mathrm{p}=0.000$ y OR $1.9 \mathrm{p}=0.000$ respectivamente). Los triglicéridos fueron significativamente elevados entre quienes consumían alcohol (OR $1.7 \mathrm{p}=0.04)$ (Tabla 3).

Se halló una frecuencia de TSH de $4.6 \mathrm{mIU} / \mathrm{L}$ o mayor en un $8.1 \%$ de la población de estudio. La prevalencia de hipotiroidismo manifiesto (TSH alta y $\mathrm{T}_{4}$ baja) fue de $1.2 \%$ y la de hipotiroidismo subclínico (TSH alta y $\mathrm{T}_{4} \mathrm{~L}$ normal) fue de $6.7 \%$.

Al comparar pacientes con hipotiroidismo manifiesto con aquellos que no lo tenían, la prevalencia de CT elevado fue mayor (50.0\% vs $31.6 \%$ ), dicha diferencia se observó más en los pacientes menores de 45 años (66.7\% vs $22.0 \%)$, la prevalencia de C-LDL de más de $130 \mathrm{mg} / \mathrm{dL}$ fue mucho mayor en el mismo grupo de edad (66.7\% vs $17.6 \%)$, dichas diferencias no fueron significativas.

En aquellos con hipotiroidismo subclínico se observaron diferencias significativas tanto para CT elevado (43.6\% vs $31.0 \%$ p: 0.04$)$, como para C-LDL elevado ( $40.0 \%$ vs $23.2 \%$ p: 0.006) y en este último la diferencia fue mayor en

\begin{tabular}{|c|c|c|c|c|c|}
\hline & $\mathbf{P T}^{\mathbf{1}}$ & Características & Prevalencia n $(\%)$ & $\mathbf{J i}^{2}(\mathbf{p})$ & OR (IC 95\%) \\
\hline $\begin{array}{l}\mathrm{CT}^{\mathrm{a}} \\
\geq 200 \mathrm{mg} / \mathrm{dL}\end{array}$ & $261(31.9)$ & $\begin{array}{l}\text { Edad grupos } \geq 45 \text { / }<45 \\
\text { Hombre / mujer } \\
\text { Años escolaridad }<8 \text { / } \geq 8 \text { años } \\
\text { Fumador } \mathrm{Si} \text { / No } \\
\text { Alcohol Si / No }\end{array}$ & $\begin{array}{l}185(37.6) / 72(22.5) \\
148(30.8) / 113(33.4) \\
197(36.5) / 56(21.5) \\
33(30.8) / 217(31.6) \\
166(30.5) / 86(33)\end{array}$ & $\begin{array}{c}<\mathbf{0 . 0 0 0} \\
0.447 \\
<\mathbf{0 . 0 0 0} \\
0.91 \\
0.37\end{array}$ & $\begin{array}{l}2.1(\mathbf{1 . 5}-\mathbf{2 . 9}) \\
0.9(0.75-1.13) \\
\mathbf{2 . 1}(\mathbf{1 . 5}-\mathbf{3 . 0}) \\
0.96(0.6-1.5) \\
0.9(0.6-1.2)\end{array}$ \\
\hline $\begin{array}{l}\text { C-HDL } \\
<40 \mathrm{mg} / \mathrm{dL}\end{array}$ & $474(57.9)$ & $\begin{array}{l}\text { Edad grupos } \geq 45 /<45 \\
\text { Hombre / mujer } \\
\text { Años escolaridad }<8 \text { / } \geq 8 \text { años } \\
\text { Fumador } \mathrm{Si} \text { / No } \\
\text { Alcohol Si / No }\end{array}$ & $\begin{array}{l}268(54.7) / 199(62.2) \\
301(62.6) / 173(51.2) \\
300(55.7) / 159(61.2) \\
124(61.4) / 339(56.2) \\
325(59.6) / 135(52.9)\end{array}$ & $\begin{array}{l}\mathbf{0 . 0 3 5} \\
\mathbf{0 . 0 0 1} \\
0.08 \\
0.11 \\
0.04\end{array}$ & $\begin{array}{l}0.7(0.6-1.0) \\
\mathbf{1 . 6}(\mathbf{1 . 2}-\mathbf{2 . 1}) \\
0.8(0.6-1.1) \\
1.2(0.9-1.7) \\
1.3(1.0-1.8)\end{array}$ \\
\hline $\begin{array}{l}\text { C-LDL } \\
\geq 130 \mathrm{mg} / \mathrm{dL}\end{array}$ & $199(24.3)$ & $\begin{array}{l}\text { Edad grupos } \geq 45 /<45 \\
\text { Hombre / mujer } \\
\text { Años escolaridad }<8 \text { / } \geq 8 \text { años } \\
\text { Fumador } \mathrm{Si} \text { / No } \\
\text { Alcohol Si / No }\end{array}$ & $\begin{array}{l}138(28.2) / 58(18.1) \\
118(24.5) / 81824) \\
150(27.8) / 44(16.9) \\
51(25.2) / 143(23.7) \\
129(23.7) / 64(25.1)\end{array}$ & $\begin{array}{r}\mathbf{0 . 0 0 1} \\
0.46 \\
<\mathbf{0 . 0 0 0} \\
0.36 \\
0.36\end{array}$ & $\begin{array}{l}\mathbf{1 . 8}(\mathbf{1 . 2}-\mathbf{2 . 5}) \\
1.0(0.7-1.4) \\
\mathbf{1 . 9}(\mathbf{1 . 3 - 2 . 7 )} \\
1.1(0.8-1.6) \\
0.9(0.7-1.3)\end{array}$ \\
\hline $\begin{array}{l}\text { TG }^{\mathrm{d}} \\
\geq 200 \mathrm{mg} / \mathrm{dL}\end{array}$ & $213(26.0)$ & $\begin{array}{l}\text { Edad grupos } \geq 45 /<45 \\
\text { Hombre / mujer } \\
\text { Años escolaridad }<8 \text { / } \geq 8 \text { años } \\
\text { Fumador } \mathrm{Si} \text { / No } \\
\text { Alcohol Si / No }\end{array}$ & $\begin{array}{l}133(27.1) / 76(23.8) \\
126(26.2) / 87(25.7) \\
134(24.9) / 74(28.5) \\
68(3.7) / 141(23.4) \\
136(25.0) / 72(28.2)\end{array}$ & $\begin{array}{l}0.16 \\
0.47 \\
0.16 \\
\mathbf{0 . 0 3} \\
0.18\end{array}$ & $\begin{array}{l}1.2(0.86-1.66) \\
1.0(0.7-1.4) \\
0.8(0.6-1.2) \\
\mathbf{1 . 7}(\mathbf{1 . 2}-2.3) \\
0.8(0.6-1.2)\end{array}$ \\
\hline
\end{tabular}


Tabla 4. Relación entre dislipidemia e hipotiroidismo.

\begin{tabular}{|c|c|c|c|c|c|c|}
\hline & \multicolumn{3}{|c|}{ Hipotiroidismo manifiesto Si / No } & \multicolumn{3}{|c|}{ Hipotiroidismo subclínico Si / No } \\
\hline & $\mathbf{P}^{1} \mathbf{n}(\%)$ & $\mathbf{J i}^{2}(\mathbf{p})$ & OR (IC 95\%) & $\mathbf{P}^{1} \mathbf{n}(\%)$ & $\mathbf{J i}^{2}(\mathbf{p})$ & OR (IC 95\%) \\
\hline \multicolumn{7}{|c|}{$\mathrm{CT}^{\mathrm{a}} \geq 200 \mathrm{mg} / \mathrm{dL}$} \\
\hline $\mathrm{n}$ total & $5(50.0) / 256(31.6)$ & 0.18 & $2.2(0.6-7.5)$ & $24(43.6) / 237(31.0)$ & 0.04 & $1.7(1.0-3.0)$ \\
\hline Edad $\geq 45$ & $3(42.9) / 186(37.9)$ & 0.53 & $1.2(0.3-5.6)$ & $17(45.9) / 172(37.3)$ & 0.19 & $1.4(0.7-2.8)$ \\
\hline Edad $<45$ & $2(66.7) / 70(22.0)$ & 0.13 & $7.1(0.6-79.0)$ & $7(38.9) / 65(21.5)$ & 0.08 & $2.3(0.9-6.2)$ \\
\hline $\mathrm{IMC}>25$ & $2(66.7) / 135(34.1)$ & 0.27 & $3.9(0.3-43.0)$ & $10(41.7) / 127(33.9)$ & 0.28 & $1.4(0.6-3.2)$ \\
\hline $\mathrm{IMC} \leq 25$ & $3(42.9) / 121(29.3)$ & 0.34 & $1.8(0.4-8.2)$ & $14(45.2) / 110(28.3)$ & 0.04 & $2.1(1.0-4.4)$ \\
\hline \multicolumn{7}{|c|}{$C-L L^{c} \geq 130 \mathrm{mg} / \mathrm{dL}$} \\
\hline $\mathrm{n}$ total & $5(50.0) / 194(24.0)$ & 0.07 & $3.2(0.9-11.1)$ & $22(40.0) / 177(23.2)$ & 0.006 & $2.2(1.3-3.9)$ \\
\hline Edad $\geq 45$ & $3(42.9) / 138(28.1)$ & 0.31 & $1.9(0.4-8.7)$ & $15(40.5) / 126(27.3)$ & 0.07 & $1.8(0.9-3.6)$ \\
\hline Edad $<45$ & $2(66.7) / 56(17.6)$ & 0.08 & $9.3(0.8-104.9)$ & $7(38.9) / 51(16.8)$ & 0.03 & $3.1(1.2-8.5)$ \\
\hline IMC $>25$ & $2(66.7) / 98(24.7)$ & 0.26 & $6.1(0.5-67.8)$ & $8(33.3) / 92(24.5)$ & 0.23 & $1.5(0.6-3.7)$ \\
\hline $\mathrm{IMC} \leq 25$ & $3(42.9) / 96(23.2)$ & 0.21 & $2.5(0.5-11.2)$ & $14(45.2) / 85(21.9)$ & 0.005 & $2.9(1.4-6.2)$ \\
\hline \multicolumn{7}{|c|}{$\mathrm{TG}^{\mathrm{d}} \geq 150 \mathrm{mg} / \mathrm{dL}$} \\
\hline $\mathrm{n}$ total & $3(30.0) / 374(46.2)$ & 0.24 & $0.5(0.1-1.9)$ & $26(47.3) / 351845.9)$ & 0.48 & $1.1(0.6-1.8)$ \\
\hline Edad $\geq 45$ & $3(42.9) / 246(50.1)$ & 0.5 & $0.7(0.2-3.4)$ & $21(56.8) / 228(49.5)$ & 0.25 & $1.3(0.7-2.3)$ \\
\hline Edad $<45$ & $0(0) / 128(40.3)$ & - & - & $5(27.8) / 123(40.6)$ & 0.21 & $0.6(0.2-1.6)$ \\
\hline $\mathrm{IMC}>25$ & $3(100) / 229(57.8)$ & - & - & $16(66.7) / 216(57.6)$ & 0.26 & $1.5(0.6-3.5)$ \\
\hline $\mathrm{IMC} \leq 25$ & $0(0) / 145(35.1)$ & - & - & $10(32.3) / 135(34.7)$ & 0.47 & $0.9(0.4-2.0)$ \\
\hline
\end{tabular}

los menores de 45 años. Así mismo, tanto en CT como en C-LDL elevados se observaron diferencias significativas en quienes tenían IMC por debajo de 25 (Tabla 4).

Se encontró relación significativa entre las TSH de 4.6 $\mathrm{mIU} / \mathrm{L} \mathrm{o}$ valores mayores con CT elevado (OR $1.9 \mathrm{p} 0.01$ ) y C-LDL elevada (OR 2.5 p 0.001) en toda la población de estudio; también se encontró que la frecuencia de C-LDL elevada se presenta 2.2 veces en personas con hipotiroidismo subclínico con relación a quienes tienen niveles normales de hormonas tiroideas (Tabla 5).

Al realizar el análisis multivariante se encontraron asociados como factores independientes a la hipercolesterolemia la edad menor a 45 años (OR 1.8 IC 95\% 1.2-2.5) y la escolaridad menor a ocho años de estudios (OR 1.7 IC 95\% 1.2-2.4); al evaluar la elevación de C-LDL siguieron apareciendo en el modelo final la edad menor a 45 (OR 1.4 IC 95\% 1.0-2.1) y la escolaridad (OR 1.6 IC 95\% 1.1-2.5) pero aparece como el factor más importante en un modelo la TSH mayor o igual a 4.6 (OR 2.2 IC 95\% 1.3-3.7) y en el otro el hipotiroidismo subclínico (OR 2.0 IC 95\% 1.2-3.6). La hipertrigliceridemia se asoció a la edad menor a 45 (OR 1.4 IC 95\% 1.1-1.9) y al IMC por encima de 25 (OR 2.6 IC $95 \%$ 1.9-3.4)
La edad menor a 45 años sobresalió como factor independiente asociado a la hipercolesterolemia, la elevación de C-LDL y a la hipertrigliceridemia con un riesgo alrededor de 1.5 veces con respecto a los mayores de esta edad; en este estudio no se valoraron hábitos alimentarios pero es posible que las personas del campo de más años tengan una dieta más sana; para el modelo multivariante se incluyó también el consumo de alcohol sin encontrarlo asociado.

De la misma manera, haber estudiado menos de siete años se asoció tanto a CT como a C-LDL elevados. Un IMC por encima de 25 se constituyó también como factor asociado únicamente a la hipertrigliceridemia.

La alteración de las hormonas tiroideas, tanto la elevación sola de la TSH a partir de $4.6 \mathrm{mIU} / \mathrm{L}$ como el hipotiroidismo subclínico resultaron ser en los modelos multivariantes el factor más importante para la elevación del C-LDL con un riesgo mayor a dos con respecto a las personas con hormonas normales.

\section{Discusión}

En el mundo, la primera causa de mortalidad son las enfermedades cardiovasculares, condicionadas muchas de ellas por cifras persistentes de lípidos altos en sangre; la literatura 
Tabla 5. Relación entre dislipidemia y alteraciones en las hormonas tiroideas.

\begin{tabular}{|c|c|c|c|c|c|c|}
\hline & \multicolumn{3}{|c|}{$\mathrm{TSH} \geq 4.6 \mathrm{Si} / \mathrm{No}$} & \multicolumn{3}{|c|}{$\mathrm{TSH} \geq 10 \mathrm{Si} / \mathrm{No}$} \\
\hline & $\mathbf{P}^{1} \mathbf{n}(\%)$ & $\mathbf{J i}^{2}(\mathbf{p})$ & OR (IC95\%) & $\mathbf{P}^{1} \mathbf{n}(\%)$ & $\mathbf{J i}^{2}(\mathbf{p})$ & OR (IC 95\%) \\
\hline \multicolumn{7}{|c|}{$\mathrm{CT}^{\mathrm{a}} \geq 200 \mathrm{mg} / \mathrm{dL}$} \\
\hline $\mathrm{n}$ total & $29(46) / 226(30.7)$ & 0.01 & $1.9(1.1-3.2)$ & $6(42.9) / 23(45.1)$ & 0.25 & $2.1(0.5-9.0)$ \\
\hline Edad $\geq 60$ & $11(50) / 60(37.3)$ & 0.18 & $1.7(0.7-4.1)$ & $4(66.7) / 7(43.8)$ & 0.32 & $2.6(0.4-18.3)$ \\
\hline Edad $\geq 45$ & $20(45.5) / 163(36.8)$ & 0.17 & $1.4(0.8-2.7)$ & $6(60) / 14(41.2)$ & 0.56 & $0.9(0.3-3.0)$ \\
\hline Edad $<45$ & $9(42.9) / 63(21.6)$ & 0.03 & $2.7(1.1-6.8)$ & $0(0) / 9(52.9)$ & - & - \\
\hline \multicolumn{7}{|c|}{$C-L L^{c} \geq 130 \mathrm{mg} / \mathrm{dL}$} \\
\hline $\mathrm{n}$ total & $27(42.9) / 168(22.8)$ & 0.001 & $2.5(1.5-4.3)$ & $6(42.9) / 21(41.2)$ & 0.57 & $1.1(0.3-3.5)$ \\
\hline Edad $\geq 60$ & $10(45.5) / 43(26.7)$ & 0.06 & $2.3(0.9-5.7)$ & $4(66.7) / 6(37.5)$ & 0.22 & $3.3(0.5-24.0)$ \\
\hline Edad $\geq 45$ & $18(40.9) / 119(26.9)$ & 0.04 & $1.9(1.0-3.6)$ & $6(60) / 12(35.3)$ & 0.15 & $2.7(0.6-11.7)$ \\
\hline Edad $<45$ & $9(42.9) / 49(16.8)$ & 0.007 & $3.7(1.5-9.3)$ & $0(0) / 9(52.9)$ & - & - \\
\hline \multicolumn{7}{|c|}{$\mathrm{TG}^{\mathrm{d}} \geq 150 \mathrm{mg} / \mathrm{dL}$} \\
\hline $\mathrm{n}$ total & $29(44.6) / 342(46.5)$ & 0.4 & $0.9(0.6-1.5)$ & $5(35.7) / 24(47.1)$ & 0.33 & $0.6(0.2 .2 .1)$ \\
\hline Edad $\geq 60$ & $10(45.5) / 78(48.4)$ & 0.49 & $0.9(0.4-2.2)$ & $3(50) / 7(43.8)$ & 0.58 & $1.3(0.2-8.4)$ \\
\hline Edad $\geq 45$ & $24(54.5) / 222(50.1)$ & 0.34 & $1.2(0.6-2.2)$ & $5(50) / 19(55.9)$ & 0.51 & $0.8(0.2-3.2)$ \\
\hline Edad $<45$ & $5(23.8) / 120(41.1)$ & 0.09 & $0.5(0.2-1.3$ & $0(0) / 5(24.9)$ & - & - \\
\hline
\end{tabular}

informa que más del $30 \%$ de la población mundial padece de una dislipidemia (25) y una causa muy importante de esta situación es la disfunción de las hormonas tiroideas $(3,4)$.

En este estudio se exploró la relación entre dislipidemias y hormonas tiroideas en trabajadores agrícolas. Aunque se ha sugerido que los agricultores por su actividad física tienen tendencia a menos alteraciones en los lípidos plasmáticos, varios estudios han demostrado que no existen diferencias entre agricultores y no agricultores $(25,26)$; sin embargo, en nuestro estudio, el $31.9 \%$ de los participantes tenían colesterol total alto; $57.9 \%$ tenían C-HDL menor a $40 \mathrm{mg} / \mathrm{dL}$, el $24.3 \%$ tenían C-LDL elevado y $26 \%$, hipertrigliceridemia, solo el $1 \%$ de los participantes recibía medicamentos para tratamiento de dislipidemias; esta cifras de dislipidemias son muy similares a las descritas para otras poblaciones de la región (27).

La prevalencia de alteraciones en los lípidos fue significativamente más alta en agricultores de 45 y más años (CT OR: 2.1; C-HDL menor a 40, OR: 1.6 y C-LDL mayor a 130 OR: 1.8). Una escolaridad menor a ocho años se asoció también con elevación del CT y C-LDL elevados (OR: 2.1 y 1.9 respectivamente). Estos cambios en los lípidos con la edad y el género también han sido evidenciados por diferentes autores (27-29), Landázuri y colaboradores mostraron en un grupo de pacientes y sus familiares consanguíneos de primer grado que el grupo de los familiares menores de 18 años tenían cifras de colesterol y colesterol LDL menores que el grupo de familiares mayores de 18 años (27). Mientras Goh, y colaboradores, encontraron diferencias de género en todos los lípidos y las lipoproteínas, de manera que los hombres tenían niveles significativamente más altos CT, triglicéridos, C-LDL y niveles más bajos de C-HDL que las mujeres (28). También encontró Goh, que aunque los niveles de lípidos y lipoproteínas en los hombres no cambiaron en los diferentes grupos de edad, los de las mujeres, especialmente TC, LDLC y TC / HDL-C, fueron significativamente altos en las de mayor edad (> 50 años) que los niveles correspondientes en las mujeres, más jóvenes entre 30-46 años (28).

En relación con la función tiroidea en el presente estudio, el $8.0 \%$ de los participantes tenían elevación de TSH; $6.7 \%$ hipotiroidismo subclínico y $1.2 \%$ con hipotiroidismo manifiesto. Estas cifras son inferiores a lo descrito por Londoño y colaboradores (30) y Builes y colaboradores (31), 18.5\% y $36.8 \%$, ambos estudios realizados en población general del Quindío y Bogotá respectivamente. Esta discrepancia podría atribuirse a que el grupo del presente estudio tiene criterios de selección diferentes que los homogeniza más que un grupo de población general. Al respecto Fardella y colaboradores (32), encontraron en un grupo de trabajadores de diferentes empresas chilenas que el $6.8 \%$ de su grupo tenía niveles séricos de hormonas tiroideas y TSH anormales, $1 \%$ tenían hipotiroidismo, $5.6 \%$ hipotiroidismo subclínico y $0.2 \%$ tenían hipertiroidismo, cifras muy similares a las descritas en el presente estudio.

Al establecer una relación entre alteraciones de lípidos y función tiroidea, se observó que en los pacientes con hipercolesterolemia, $11.4 \%$ tenían TSH elevada $(\mathrm{p}=0.017)$ (manifiesto) y $9.2 \%$ hipotiroidismo subclínico $(\mathrm{p}=0.039)$. 
En otros trabajos se ha demostrado una asociación entre hipercolesterolemia y disfunción tiroidea, observando que individuos con esta dislipidemia, tienen prevalencias de alteraciones tiroideas variables entre 1.4 y $13 \%$ (3-5).

Esta relación posiblemente se fundamenta en el efecto que tienen las hormonas tiroideas sobre el metabolismo de los lípidos; así, el T3 se requiere para el metabolismo del colesterol y C-LDL, por el control que ejerce esta hormona en la expresión del gen que codifica el receptor $(1,33)$. Debido a esto la persistente y progresiva disminución en los niveles circulantes de hormona tiroidea, eleva en gran medida la concentración de colesterol y triglicéridos (1). Tan KC y colaboradores (34), demostraron que las subfracciones de HDL también están afectadas por la disfunción tiroidea; mientras la subfracción HDL2, estaba reducida en pacientes con hipertiroidismo, esta misma fracción estaba aumentada en pacientes con hipotiroidismo. El tratamiento de ambos tipos de pacientes revirtió estos hallazgos. Se cree que los cambios en las subfracciones de HDL son mediados por el efecto de la hormona tiroidea en la lipasa hepática (34).

Llamó la atención que se encontraran valores significativamente elevados de CT y C-LDL en participantes con hipotiroidismo subclínico y que dichas diferencias fuesen más marcadas por debajo de los 45 años de edad; dicho comportamiento no fue tan evidente en el hipotiroidismo manifiesto. Las diferencias en el CT y C-LDL fueron muy evidentes y significativas en pacientes con la TSH por encima de 4.6 $\mathrm{mIU} / \mathrm{L}$ mas no cuando la TSH tenía valores de 10, o mayores, es posible que haya sido porque en esta última el número de pacientes era muy bajo. El hipotiroidismo subclínico se caracteriza por niveles séricos elevados de TSH y niveles normales de T3 y T4 libres; es común en la población general, en especial en mujeres mayores (35). Varios autores han sugeridos una asociación entre hipotiroidismo subclínico y enfermedad cardiovascular $(23,35)$, pero esto aún es controversial, si bien algunos datos sugiere que pacientes con THS $>$ de 10 $\mathrm{mU} / \mathrm{L}$, pueden tener incrementado el riesgo cardiovascular (35), el estudio de Nair y colaboradores en población hindú, no encontró asociación entre el hipotiroidismo subclínico o el aumento de TSH y el riesgos cardiovascular a 10 años usando la escala de Framingham (36); el mismo estudio no observó diferencia entre los grupos con respecto al perfil lipídico, pero sí encontró que una TSH en aumento se correlacionó significativamente con un moderado empeoramiento del perfil lipídico (36), sugiriendo estos estudios que la TSH ejerce por sí sola un efecto independiente sobre el metabolismo de los lípidos (23). Aunque en varios trabajos se ha demostrado una fuerte asociación entre TSH y triglicéridos $(6,21)$, la asociación entre TSH y colesterol aun es controversial (37, 38); sin embargo, nuestro trabajo encontró asociación causal entre TSH elevada e hipercolesterolemia (OR 1.9) y C-LDL elevada (OR 2.5) y esta última está elevada 1.2 veces más en personas con hipotiroidismo subclínico.

La alteración de las hormonas tiroideas, tanto la elevación sola de la TSH a partir de $4.6 \mathrm{mIU} / \mathrm{L}$ como el hipotiroidismo subclínico resultaron ser en los modelos multivariantes el factor más importante para la elevación del C-LDL con un riesgo mayor a dos con respecto a las personas con hormonas normales.

La literatura describe que los pacientes con valores de TSH entre 5.1 y $10 \mathrm{mIU} / \mathrm{L}$ tienen colesterol total y C-LDL en promedio significativamente mayor en comparación con los sujetos eutiroideos (39); los mecanismos por los cuales esto ocurre están en investigación, pero hay consenso en que la dislipidemia es causada principalmente por un desbalance hacia un aumento en la síntesis de colesterol sobre la tasa de degradación, lo que lleva a la inhibición del receptor LDL y al aumento de esta lipoproteína en plasma, que proporciona así el sustrato para la peroxidación de lípidos por especies reactivas de oxígeno (ROS), lo que resulta en estrés oxidativo y puede llevar a enfermedad cardiovascular $(39,40)$.

La asociación de C-LDL con los niveles de TSH anormales refrenda la necesidad de vigilancia y control no solo del hipotiroidismo manifiesto sino del hipotiroidismo subclínico; las diferentes asociaciones relacionadas de especialistas recomiendan el tamizaje de hipotiroidismo en pacientes con dislipidemia desde antes de iniciar el tratamiento (3) con el objeto de contribuir a la disminución de la morbimortalidad por eventos cardiovasculares.

En los trabajadores del agro, por sus muy particulares condiciones socioeconómicas, es importante diseñar programas de tamizaje de biomarcadores que permitan avizorar de manera precoz cambios en las hormonas tiroideas, TSH y perfil lipídico, con el fin de implementar las medidas médicas pertinentes en cada caso.

\section{Conclusión}

Se encontró una relación entre disfunción tiroidea y lípidos plasmáticos, la alteración tiroidea, elevación sola de la TSH e hipotiroidismo subclínico resultaron ser en los modelos multivariantes el factor más importante para la elevación del C-LDL.

\section{Agradecimientos}

A los agricultores y sus familias participantes del estudio.

\section{Financiación}

Este trabajo fue financiado por el Departamento Administrativo de Ciencia y Tecnología de Colombia (Colciencias) convenio 1113_569_33303 contracto 401 of 2013 y la Universidad del Quindío proyecto interno \# 648 de 2013.

\section{Referencias}

1. Rizos CV, Elisaf MS, Liberopoulos EN. Effects of thyroid dysfunction on lipid profile. Open Cardiovasc Med J. 2011; 5: 76-84.

2. Chin CY, Ima-Nirwana S, Mohamed IN, Aminuddin A, Johari MH, Zurinah W, et al. The Relationships between Thyroid Hormones and Thyroidstimulating Hormone with Lipid Profile in Euthyroid Men. Int J Med Sci. 2014; 1: 349-55.

3. Willard DL, Leung AM, Pearce EN. Thyroid function testing in patients with newly diagnosed hiperlipidemia. JAMA Intern Med. 2014; 174: 287-89.

4. Zhang Y, Lu P, Zhung $L$, Xiao X. Association between lipids profile and thyroid parameters in euthyroid diabetic subjects: a cross-sectional study. BMC Endocr Disord. 2015; 15:12.

5. Melpomeni P, Betsi G, Dimitriadis G. Lipid abnormalities and cardiometabolic 
risk in patients with overt and subclinical thyroid disease. Hindawi Publishing Corporation J. Lipids. 2011; Article ID 575840, 9 pages.

6. Wang CY, Chang TC, Chen MF. Associations between subclinical thyroid disease and metabolic syndrome. Endocr J. 2012, 59: 911-17.

7. Sun X, Sun Y, Li WC, Chen CY, Chiu YH, Chien HY, et al. Association of Thyroid-stimulating Hormone and Cardiovascular Risk Factors. Intern Med. 2015; 54: $2537-44$

8. Boone LR, Niesen MI, Jaroszeski M, Ness GC. In vivo identification of promoter elements and transcription factors mediating activation of hepatic HMG-CoA reductase by T3. Biochem Biophys Res Commun. 2009; 385(3): 466-71.

9. Shin DJ, Osborne TF. Thyroid Hormone Regulation and Cholesterol Metabolism Are Connected through Sterol Regulatory Element-binding Protein-2 (SREBP-2). J. Biol Chem. 2003; 278: 34114-34118.

10. Pearce EN. Update in lipid alterations in subclinical hypothyroidism. J Clin Endocrinol Metab 2012; 97: 326-33.

11. Triolo M, Kwakernaak AJ, Perton FG, de Vries R, Dallinga-Thie GM, Dullaart RPF. Low normal thyroid function enhances plasma cholesteryl ester transfer in Type 2 diabetes mellitus Atherosclerosis, 2013; 228(2): 466-71.

12. Duntas LH, Brenta G. The Effect of Thyroid Disorders on Lipid Levels and Metabolism. Med Clin N Am. 2012; 96: 269-81.

13. Lu S, Guan Q, Liu Y, Wang H, Xu W, Li X, et al. Role of extrathyroidal TSHR expression in adipocyte differentiation and its association with obesity. Lipids health and dis. 2012; 11: 7.

14. Gagnon A, Antunes TT, Ly T, Pongsuwan P, Gavin C, Lochnan HA et al. Thyroid-stimulating hormone stimulates lipolysis in adipocytes in culture and raises serum free fatty acid levels in vivo. Metabolism 2010; 59: 547-53.

15. Tian L, Song Y, Xing M, Zhang W, Ning G, Li X, et al. A novel role for thyroidstimulating hormone: Up-regulation of hepatic 3-hydroxy-3-methyl-glutarylcoenzyme a reductase expression through the cyclic adenosine monophosphate/ protein kinase $\mathrm{A} /$ cyclic adenosine monophosphate responsive element binding protein pathway. Hepatology. 2010; 52: 1401-09.

16. Spadafranca A, Cappelletti C, Leone A, Vignati L, Battezzati A, Bedogni G, et al. Relationship between thyroid hormones, resting energy expenditure and cardiometabolic risk factors in euthyroid subjects. Clinical Nutrition. 2015; 34: 674-78

17. Roos A, Bakker SJL, Links TP, Gans ROB, Wolffenbuttel BHR. Thyroid Function Is Associated with Components of the Metabolic Syndrome in Euthyroid Subjects. J Clin Endocrinol Metab. 2007; 92: 491-96.

18. Garduno-García J de J, Alvirde-García U, López-Carrasco G, Padilla Mendoza ME, Mehta R, Arellano-Campos O, et al. TSH and free thyroxine concentrations are associated with differing metabolic markers in euthyroid subjects Eur J Endocrinol. 2010; 163: 273-78.

19. Åsvold BO, Vatten LJ, Nilsen TIL, Bjøro T. The association between TSH within the reference range and serum lipid concentrations in a population-based study. The HUNT Study Eur J Endocrinol. 2007; 156: 181-86.

20. Kim BJ, Kim TY, Koh JM, Kim HK, Park JY, Lee KU, et al. Relationship between serum free T4 (FT4) levels and metabolic syndrome (MS) and its components in healthy euthyroid subjects. Clin Endocrinol 2009; 70:152-60.

21. Ruhla S, Weickert MO, Arafat AM, Osterhoff M, Isken F, Spranger J, et al. A high normal TSH is associated with the metabolic syndrome. Clin Endocrinol. 2010; 72: 696-01.

22.Saleem MS, Shirwany TAK, Khan KA. Relationship of thyroid-stimulating hormone with metabolic syndrome in a simple of euthyroid Pakistani population J Ayub Med Coll Abbottabad. 2011; 23: 63-8.

23. Wang F, Tan Y, Wang C, Zhang X, Zhao Y, Song X, et al. Thyroid-Stimulating Hormone Levels within the Reference Range Are Associated with Serum Lipid Profiles Independent of Thyroid Hormones. J Clin Endocrinol Metab 2012; 97: 2724-31.

24. Friedewald WF, Levy RI, Fredrickson DS. Estimation of the concentration of low-density lipoprotein cholesterol in plasma without use of the preparative ultracentrifuge. Clin Chem. 1977; 18: 499-502.

25. Prokosch AJ, Dalleck LC, Pettitt RW. Cardiac Risk Factors between Farmers and Non-Farmers. J Exerc Physiol-online 2011; 14: 91-00.

26. Jenkins P, Earle-Richardson G, Bell J, May J, Green A. Chronic disease risk in central New York dairy farmers: Results from a large health survey 1989-1999 Am J Ind Med. 2005; 47: 20-26

27. Landázuri P, Loango N, Gallego ML. Cardiovascular risk factors in first-degree relatives of patients with hypertension Colomb Med. 2011; 42: 17-25.

28. Goh V, Tong T, Mok H, Said B. Differential impact of aging and gender on lipid and lipoprotein profiles in a cohort of healthy Chinese Singaporeans. Asian J Androl. 2007; 9: 787-94.

29. Anagnostis P, Stevenson, JC, Crook D, Johnston DG, Godsland IF. Effects of menopause, gender and age on lipids and high-density lipoprotein cholesterol subfractions Maturitas. 2015; 81: 62-68.

30. Londoño AL, Gallego ML, Bayona A, Landázuri P. Prevalencia de hipotiroidismo y relación con niveles elevados de anticuerpos antiperoxidasa y yoduria en población de 35 y más años en Armenia. 2009-2010 Rev Salud Pública. 2011; 13: 998-09.

31. Builes CA, Rosero O, García J. Evaluación de disfunción tiroidea según TSH en una población de Bogotá. Acta Med Colomb. 2006; 31: 66-70.

32. Fardella C, Poggi H, Gloger S, Rojas A, Velásquez CG, Barroileth S et al. High prevalence of subclinical thyroidal disease among individuals attended in occupational health examinations Rev med Chil. 2001; 129: 155-160.

33. López D, Abisambra Socarrás JF, Bedi M Ness GC. Activation of the hepatic LDL receptor promoter by thyroid hormone. Biochim Biophys Acta. 2007; 1771:1216-25

34. Tan KC, Shiu SW, Kung AW. Effect of thyroid dysfunction on high-density lipoprotein subfraction metabolism: Roles of hepatic lipase and cholesteryl ester transfer protein J Clin Endocrinol Metab. 2013; 83; 2921-24.

35. Decandia F. Risk factors for cardiovascular disease in subclinical hypothyroidism. Ir J Med Sci, 2017 DOI 10.1007/s11845-017-1617-9

36. Nair SN, Kumar H, Raveendran M, Menon VU. Subclinical Hypothyroidism and Cardiac Risk: Lessons from a South Indian Population Study. Indian J Endocrinol Metab 2018; 22(2): 217-222.

37. Delitala AP, Fanciulli G, Maioli M Delitala G. Subclinical hypothyroidism, lipid metabolism and cardiovascular disease. Eur J Intern Med, 2017; 38: 17-24.

38. Díez JJ, Iglesias P. Serum cholesterol and triglyceride levels in diabetic patients with subclinical hypothyroidism. Endocrinol Nutr 2014; 61:419-25.

39. Duntas LH, Brenta G.A Renewed focus on the association between thyroid hormones and Lipid Metabolism. Front Endocrinol 2018; 9: doi: 10.3389/ fendo.2018.00511

40. Gong Y, Ma Y, Ye Z, Fu Z, Yang P, Gao B, et al. Thyroid stimulating hormone exhibits the impact on LDLR/LDL-c via up-regulating hepatic PCSK9 expression. Metabolism clinical and experimental 76 (2017) $32-41$ 